\title{
Managing closure risks by integrating acid and metalliferous drainage assessments with mine scheduling - real world applications
}

\author{
S.R. Pearce GHD Pty Ltd, Australia \\ F.R. Beavis GHD Pty Ltd, Australia \\ S.J. Winchester GHD Pty Ltd, Australia \\ H. Thompson GHD Pty Ltd, Australia
}

\begin{abstract}
Acid and metalliferous drainage (AMD) impacts may be a cause of significant long term environmental liabilities, as evidenced on many historic mine sites containing legacy AMD issues worldwide. These historical precedents have led to AMD being recognised as a key closure risk by industry and regulators, which in turn has driven progressive advances in geochemical assessment and management in recent times.
\end{abstract}

Many AMD risks on a typical mine site are based on mineral waste management strategy and practice. By association, mine planning and scheduling may therefore have a significant bearing on the potential for $A M D$ related closure liabilities. Despite this, the assessment of AMD risk is often packaged into the environmental approvals and management process, which is often not directly connected with the mine planning and scheduling process. Consequently, AMD assessments and management plans are frequently progressed by an approvals team that is often somewhat disconnected from the mine planning and scheduling team. Subsequently, AMD management measures may be conceived after the scheduling process has moved into a more advanced and less flexible phase. Sometimes, this may be the result of a lack of early co-ordination and/or budget leading to a lack of hard geochemical data with which to begin classifying potentially acid forming material.

Using an integrated approach to managing closure risk can be achieved via a method of assessment utilising 3D geochemical block modelling that can operate concurrently with (or within) the mine planning and scheduling process from early in the mine planning process. A more integrated approach sees AMD assessment and management being advanced concurrently with resource modelling to optimise results and minimise risk.

Three case studies are presented in this paper where an integrated approach was used for conceptual closure planning through the environmental approvals stage. A further case study is presented where an integrated approach was taken to develop a mine plan. The application of this approach has so far proven encouraging.

\section{Introduction}

The issue of AMD arguably remains one of the largest threats to realising long-term environmental sustainability within the mining industry. Ecological and financial implications resulting from long-term AMD is a global problem, with impacts primarily affecting surface waters and their associated ecosystems (Harries, 1997; Mudder, 2004).

One industry standard method to manage and mitigate the AMD risk is by material characterisation, that is, by generating a sound understanding of the deposit geology and geochemistry through early geochemical characterisation. However, as the mine environmental impact assessment (EIA) process, which may include a conceptual closure plan incorporating a mine waste geochemical assessment commensurate with 
regulatory requirements, is often undertaken during mine scoping or pre-feasibility; this approach is not always achievable. Reasons may include funding limitations at the early mine planning stage and/or a focus on defining mineable reserves such that the exploration drilling budget is allocated to characterising ore, rather than waste; the latter required to determine a large component of the forward AMD risk.

Commensurate with the geochemistry of a deposit, and its subsequent development, any AMD potential may remain a latent risk only realised over future generations. Such risk is fundamentally opposed to the concept of sustainability and inter-generation equity within the mining industry, in addition to be tangential to several of the 10 Principles as defined under the International Council for Mining and Metals' (ICMM) Sustainable Development Framework (Bruntland, 1987; ICMM, 2003).

It is therefore logical that effectively co-ordinating any limited budgets between the mine planning, geology and environment departments at the early mine planning stage is a sound strategy. This approach seeks to maximise the return on investment to reduce forward AMD risk over the long term. There are several leading practice documents within the public domain that advocate such a strategic approach, particularly between exploration, mine planning and environment teams (DITR, 2007, Section 3.1; INAP, 2009, Chapter 4).

Furthermore, any initial early stage coordinated geochemical sampling and analysis completed in coordination with other departments represents only a basic starting point for ongoing life of mine AMD risk management from a mine waste characterisation perspective. Often due to a combination of limited sample numbers due to financial limitations as noted above, in addition to quality assurance issues as they relate to sample provision and quality (e.g reverse circulation drilling chips rather than diamond core, retrospective core shed sampling following core oxidation etc); early mine life geochemical samples may not be entirely representative of forward in situ conditions. Therefore, logic suggests that a coordinated strategic approach to minimising future AMD risk through collaboration between geology, environmental and mine planning departments is a smart way to maximise the initial return on investment pending ongoing life of mine sampling and analysis to increase statistical confidence, and therefore, further lower risk.

Notwithstanding the growing global body of knowledge on AMD through industry collaboration via global bodies such as The International Network for Acid Prevention (INAP) for example, there remains much to add to the knowledge base. It is generally recognised that this collegiate approach is the way forward; this paper provides a real world example of how a collaborative project based approach was achieved for three case study projects.

\section{$2 \quad$ AMD risk and early mine closure planning}

As the global body of AMD knowledge grows, with recognition of the associated risks, regulators are increasingly incorporating AMD specific requirements into the mine environmental approvals process. One mechanism to achieve this beyond the standard, generalist Environmental Impact Assessment (EIA) process is via an increased requirement for detail in the preliminary mine closure plan for submission with the relevant environmental approvals document.

There remain several examples of tightening mine closure legislation and guidelines including for example Chile and Western Australia (WA). In WA, updated guidelines introduced in 2011 require robust mine closure plans to be developed before environmental approval is granted (Western Australian Department of Mines and Petroleum and the Western Australian Environmental Protection Authority, 2011). These mine closure planning requirements have a significant focus on potential AMD risk, and in particular, understanding the geochemistry of the material through appropriate mine waste characterisation, with subsequent management and mitigation strategies defined as appropriate. Closure risks with respect to AMD, therefore, must be clearly assessed at the planning stage. Ultimately, a clear regulatory message is emerging inferring that unless there is a demonstrable informed plan and process to close the mine, it will not be opened. This message is being supplemented with environmental/rehabilitation bonds now approximating 100 percent of calculated rehabilitation costs; whereby historically they were arguably 
approaching tokenism. Such a paradigm shift potentially reflects increased commitment by the industry to acknowledge community expectations, and therefore, maintain a social licence to operate; itself increasingly recognised as a leading corporate risk.

The sheer scale of modern mining operations resulting from technological improvements, themselves driven by a need to maximise economies of scale to turn sub-economic opportunities into economic mines, is seeing unprecedented tonnages of mine waste being managed. One only needs to look to the Pilbara or Gallillee Basin to appreciate the scale of operations being brought on line. Using a simple linear extrapolation by tonne of waste handled and stored, AMD risk is subsequently increased over the life of mine and beyond. The concept of early coordinated assessment to inform the mine waste management strategy and subsequent management plan, is therefore becoming increasingly critical. Simple multiplication demonstrates an increased cost for retrospective materials handling to remediate AMD should initial mine planning consider the risk and manage it accordingly through selective handling and placement based on sound science.

\section{$3 \quad$ AMD - current leading practice}

As alluded to above, a considerable knowledge base has been developed on AMD and its characterisation, management, and treatment; much of it driven by the mining industry itself to better understand and manage that risk. For example, the International Network for acid Prevention (INAP) was formed in 1998 by industry to provide a technology transfer platform for sharing AMD information. In 2009, INAP released the Global Acid Rock Drainage Guide (the GARD Guide); a document that aimed to consolidate much of the global AMD knowledge base into a usable document. The information consolidated into the GARD Guide was sourced from several international research initiatives including for example the Minesite Environment Neutral Drainage (MEND) initiative (refer www.mend-nedem.org) and the previous Australian Centre for Minesite Environmental Research (ACMER), now incorporated into the Sustainable Minerals Institute (SMI) Knowledge Transfer (refer www.jktech.com.au and/or www.cmlr.uq.edu.au).

At a more local level, the Australian Commonwealth Government, in association with industry and private bodies developed a series of 'leading practice' booklets; several of which are directly applicable to managing AMD risk within the concept of mine EIA, operations and closure.

The initiative was titled 'Leading Practice Sustainable = Sustainable Development for The Mining Industry' (refer www.ret.gov.au/resources/resources_programs/lpsdmining/Pages/default.aspx).

As articulated in the reference material cited above, the clear message to best manage AMD risk is to work collaboratively across business units from exploration through to mine closure. There is now arguably a clear business model that demonstrates that planning for closure from exploration is cost effective, and therefore, desirable. This model excludes intangibles such as brand and reputational risk, and regulatory intervention. That notwithstanding, planning for closure is arguably now a well established concept in the industry.

More specifically with regard to materials characterisation to mitigate forward AMD risk, a summation of thoughts from the international leading practice documents noted above regarding the timing of AMD assessments within the mine planning process is:

- Exploration drilling (pre-feasibility): Basic geochemical and metallurgical test work are used as indicators for AMD. Stage 1 Assessment is carried out to identify potential AMD risks.

- Resource definition drilling (pre-feasibility): At this point there should be a reasonable idea of pit shell, potential waste and ore tonnages, and importantly mineralogy and geochemistry. More detailed and targeted Stage 2 AMD testing is to be carried out at this point.

- Approvals stage (may be at either of the above stages): Adequate information should be provided to produce a mine closure plan that considers potential AMD issues and adequately addresses any potential AMD risks. 
- Post approvals: Further detailed AMD testing is to include new drill holes and testing of stored core (kinetic tests) to refine geochemical assessments.

- Prior to submitting mining proposal: A detailed AMD management plan should be produced which adequately addresses how reactive materials are to be managed during mine operation, and includes detailed design for waste storage solutions and post closure monitoring plan.

Life of mine AMD monitoring would also be undertaken including surface and ground water, in addition to tailings and mine waste to increase confidence in the data set, and therefore, lower forward risk and increase regulator and community confidence.

\section{$4 \quad$ Mine planning in context}

In the context of this discussion of AMD assessment and mine closure, there are a number of aspects of mine planning that are relevant. These are:

1. Mine planning is not a simply agreed and consistently applied discipline across the mining community. It is different for each mine, and different in the particular phase of project development relevant to a specific point in time. Of real note here is that each mine does need a bespoke 'mine planning process' created for it, to serve its' specific needs. However very few mines, be they operations or projects, really do plan this vital activity as a process. Often mine planning 'just happens'.

2. Modern Mine Planning is largely done in the three context horizons. These being strategic, tactical and production planning. These should be vertically and horizontally integrated, but at times are not. The focus of this paper is really on the strategic and upper tactical planning. Although it is worth noting that if activities do not make it through to the production planning cycle, then they actually do not get completed. Therefore understanding the timing and changing focus of mine plans is quite important, especially if integration of disciplines and influence in the process is sought. It is also worth understanding that these are usually happening simultaneously - at least on producing mine sites.

3. Modern mine planning, across all horizons, is characterised by:

a. Targets: what we need to achieve, for this plan.

Objectives: the characteristics of what that successful achievement is. For example grade control, or cost characteristics.

Restrictions: what are the rules that need to be conformed to? These are best expressed in a prioritised manner.

Outcomes: what needs to be shown, to gain acceptance that the plan is valid. This needs to include an assessment of resources and costs required to achieve the stated plan.

This is the common area of failure for AMD assessment in mine planning. In that, whatever success actually is (in managing AMD on the particular project), it is not included as a criteria on how we judge the mine plan. Therefore it has little impact in how the mine plan is put together. One outcome of this is that resources, in terms of equipment and priorities, are then not assigned to AMD compliance. Therefore it (AMD control) doesn't happen as an integrated component of operations.

b. A general lack of successive guidelines as outcomes is a modern characteristic. One of the hallmarks of best practice in mine planning is that the mine plan at a higher level, say at Life of Asset, does produce clear planning guidelines for the successful planning at the subsequent (shorter time interval) level. In the AMD context this may be that the next level of planning (say at five year plan level, after the LOM), should be saying "Show that sufficient quantities of inert waste are available, each quarter, to provide for the on-going capping of the PAF waste generated as a Run of Mine material". 
4. Guidelines for AMD compliance are rarely expressed in a manner in which they can be incorporated into the design of the mine planning process.

\section{$5 \quad$ The environmental approvals and mine planning processes, and any consequences from a disconnect}

Whilst not always the case, there remains the potential for a knowledge transfer disconnect to exist between the environmental approvals team, the mine planning team, and the exploration team. This may occur commensurate with the resources used on a project specific basis; i.e. three independent consulting companies working in parallel, yet interfacing only through a Client side Project Development Manager for example; particularly at scoping or pre-feasibility stage. Transfer of AMD risk within the context of pit optimisation and mine waste management through selective handling and storage therefore becomes a function of an efficient and effective communications protocol. Such an arrangement brings its own project management risks, which ultimately, may result in a long term AMD liability as a function of its application.

Figure 1 is a schematic that attempts to demonstrate the three key project teams operating in parallel throughout pre-feasibility, yet also largely in isolation, as noted above. Although somewhat simplistic in nature, the concepts are arguably relevant to managing latent AMD risk throughout the life of mine as there remains a temporal lag between the approvals and environment team's involvement with the geological modelling and exploration team's involvement. Again, whilst not always so, such a scenario is often financially driven and/or due to a lack of holistic process understanding.

Whilst team separation as illustrated in Figure 1 may potentially be understandable given each team's individual focus, potentially based on schedule and budget, a common goal, being project development, should ideally drive information transfer through planned pathways to maximise return on investment as noted above.

The key risks by not sharing intelligence across business units at the pre feasibility stage is that of rework, ultimately costing time, and therefore, an opportunity cost of production which ultimately translates to money. A disjointed submission to regulatory agencies for approval ultimately erodes confidence and brings with it brand and reputational risk; potentially more damaging than the immediate technical AMD risks.

An additional risk from the disconnect shown in Figure 1 is a potential lack of synthesis between the more advanced mine engineering design, and the lag in geochemical data that intuitively should inform that design. An example potentially being a lost opportunity to innovatively manage potentially acid forming (PAF) mine waste through offsetting virgin material for run of mine (ROM) pad core construction material which would also act as an oxidation mitigating strategy for that PAF material and/or its use as paste backfill underground; commensurate with the mine scenario. Retrospectively redesigning engineering and/or tweaking early works costs time and money, as noted above.

Examples of some additional, potentially disadvantageous scenarios arising from the situation as shown in Figure 1 include:

- The geological block model used for resource modelling does not contain geochemical data. The implications may be dual data sets and potential management issues.

- Samples of non ore material external to the ore body being discarded, i.e. a lost data point for mine waste characterisation.

- A non representative data set for retrospective mine waste characterisation as sampled from the core shed.

- Miss-classification of construction material.

- An underestimation of 'at risk' mine waste material leading to inaccurately designed mine waste storage structures, and the AMD mitigation strategies thereof. 
Planners and schedulers

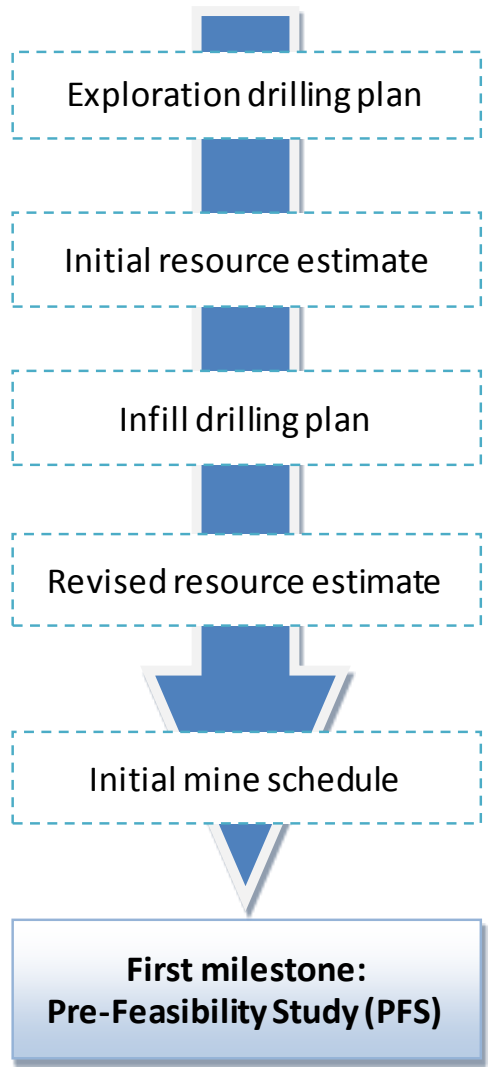

Project based mining studies

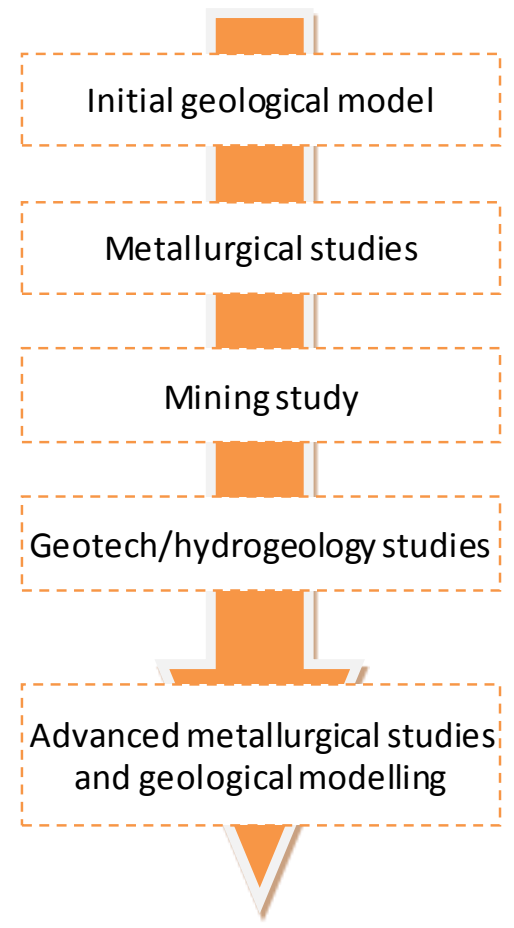

Approvals/environment team

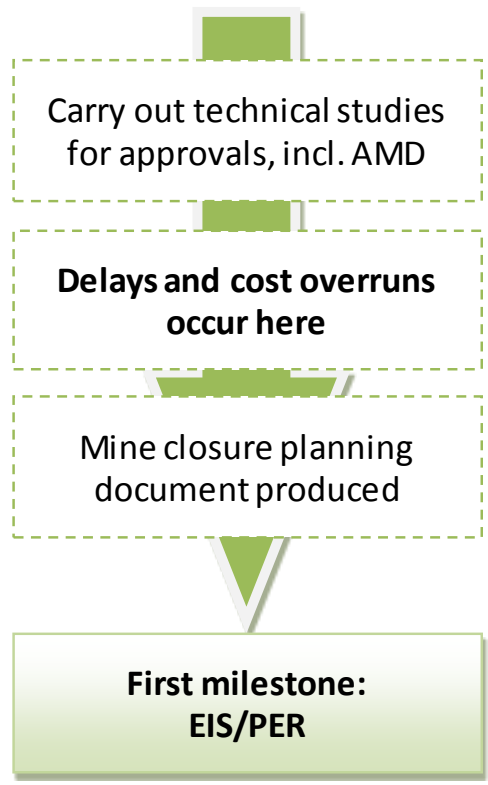

\section{Figure 1 Team schematic for a conceptual mine's pre-feasibility stage}

\section{The integrated approach}

Figure 1 presented the scenario whereby the three key mine project development teams were working largely in parallel, yet in isolation. Figure 2, on the other hand, presents an idealised scenario whereby all three key project development teams are aligned and iterative.

As noted in Section 3 of this paper, global leading practice documentation suggests an increasing geochemcial dataset from exploration through to operations and closure such that the balance of cost versus risk is appropriately maintained. This balance is ultimately managed through risk, itself ascertained by accepted statistical techniques such as confidence limits and variography, commensurate with the commodity, deposit geology, internal and external standards, and statutory and regulatory requirements.

Put simply, early investment in geochemical characterisation to better understand the risk and subsequently inform mine waste handling and long term management adds significant value throughout the life of mine and beyond. The initial dataset is then built upon to lower forward risk through increased statistical confidence. That is, a dynamic and iterative ongoing sampling and analysis plan is developed for the life of mine to validate the initial dataset and manage ongoing risk.

Once a suitable geochemical dataset has been established, the information can be modelled using a 3D software tool that integrates with mine planning software. Such an approach may ensure that the pit optimisation modelling and mine scheduling being undertaken to ensure the most cost effective resource recovery scenario includes any limitations such as potentially acid forming (PAF) material for example. Importantly, the geochemical model and the pit optimisation model are compatible ensuring that the knowledge base is maximised throughout the mine planning phase. There are clear benefits to this model, which add weight to aspiring to the scenario as shown in Figure 2. 


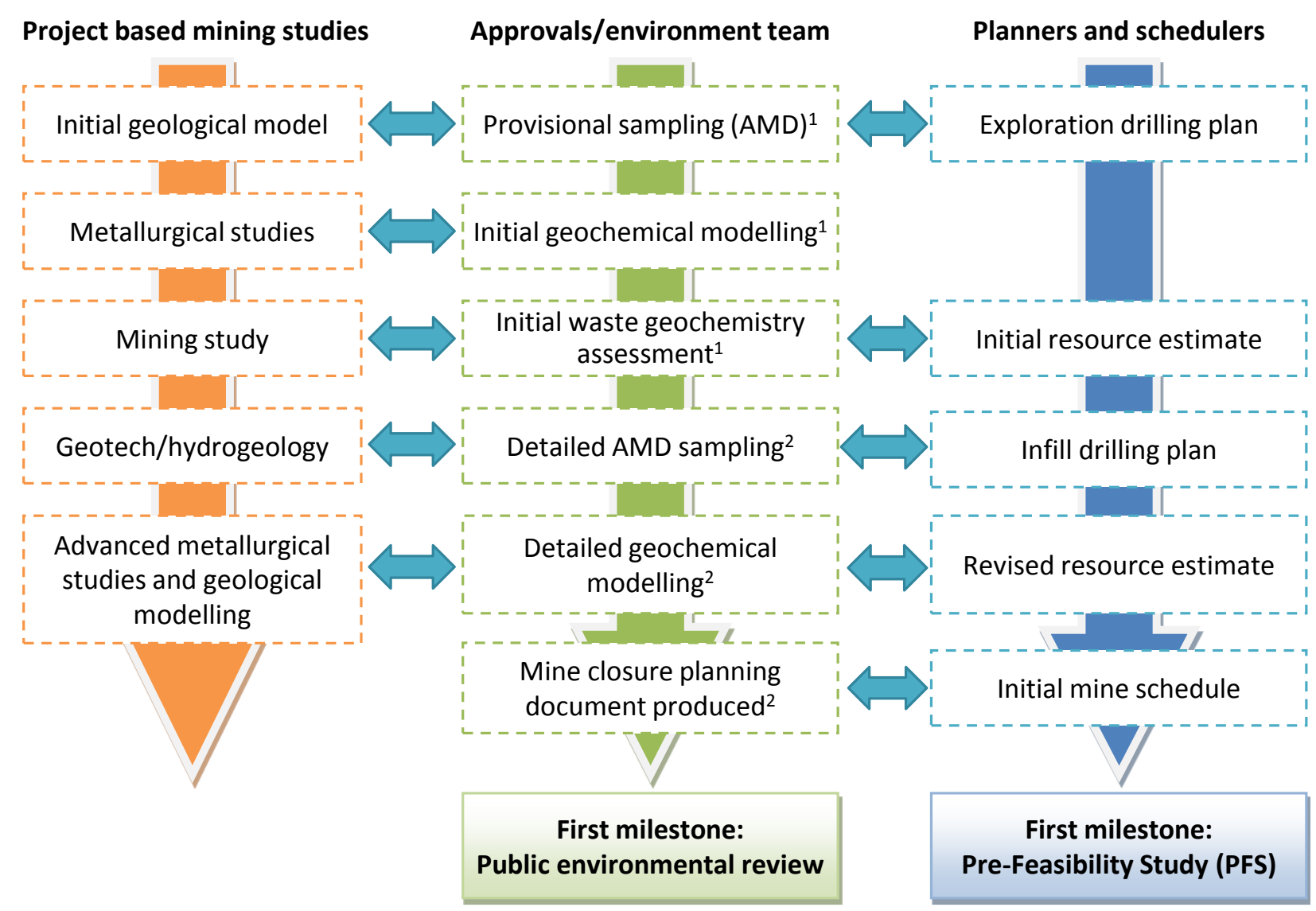

\section{Figure 2 An integrated approach}

\footnotetext{
${ }^{1}$ Denotes Stage 1 analysis

${ }^{2}$ Denotes Stage 2 analysis
}

The goals of AMD assessment require a great deal of effort and technical assessment in many cases. Geochemical characterisation is the primary assessment factor that impacts all AMD considerations and project risks, and in the context of progressive assessment should be carried out in multiple stages. Figure 2 indicates a two stage process which complements the early stages of the mine planning process:

Stage 1: High level assessment of drill hole data base which can be completed during exploration drilling and initial resource definition phase.

Stage 2: Detailed test-work program to verify the high level assessment which can be completed prior to initial mine schedule being produced, often during infill drilling programs.

The goal of Stage 1 is to identify the likely physical and geochemical and mineralogical properties (long term stability) of waste and ore materials. Ultimately the goal of Stage 1 is to produce a high level AMD risk assessment which will define the scope for the detailed AMD test work program (Stage 2).

Stage 2 works as outlined in Figure 2 involve the production and implementation of a detailed test work program. The advantages of completing many of the Stage 2 works within the infill drilling phase become apparent when the nature of geochemical test work is related to the mine planning process:

- Sufficient fresh material must be collected for testing purposes. Depending on the results of the Stage 1 analysis this may include the requirement to collect hundreds of samples from many locations. The infill drilling programme allows for the most economical and timely provision of materials for this exercise to be executed.

- The requirement for kinetic testing is best established at an early stage as results from testing may take 6-12 months to obtain. Results may fundamentally alter assumptions regarding the geochemical nature of the materials in the project area and may redefine AMD risks and 
therefore mine and closure planning requirements. It is therefore advantageous to establish a kinetic test work program as soon as possible in the planning process.

- There is potentially a positive feedback loop between sampling and the requirement for more sampling. Testing may indicate that initial geochemical classifications of materials are incorrect, as a result additional samples and testing may be required to provide additional information. This requires a means to obtain sample material in a cost effective and timely manner.

The production of the detailed geochemical model concurrently with the revised resource estimate is important to ensure that the resource model accounts for limitations that may be imposed by AMD risks that are coded within the geochemical model. The geochemical model is produced in a format that is compatible with the geological model and therefore can be integrated with ease.

Key stages of the AMD assessment involve the linkage of the mine closure plan with mine planning and scheduling. The advantages of robust AMD assessment prior to this point in time include:

- Robust materials management can be built into the schedule, i.e. haul distances or waste materials can be defined based on the requirements for in pit or ex pit disposal.

- Restrictions on mine depth or extent due to AMD issues such as dewatering risks can be built into the plan.

Timelines for the production of AMD material can be established. It is important to establish for example if a high proportion of high risk material will be extracted within a specific year as provision will need to be made for management within this timeframe (for example ensuring adequate encapsulation material is available).

Three case studies are presented that demonstrate how involving the environment team for early stage AMD assessment as part of the approvals process has proven a success by their iterative involvement with the exploration and mine planning teams.

\subsection{Case study 1 - Pilbara, WA}

A preliminary AMD assessment was completed for a greenfields site during the environmental planning process for a proposed iron ore mine. The initial desktop assessment which interrogated the total sulfur data set within the geological database identified the presence of some PAF material.

The desktop assessment indicated that the majority of the PAF material was present as the well documented Mt McRae shale unit. Fortunately, this unit was modelled to be located stratigraphically below the main ore body, and therefore, below the modelled pit shell. The vertical separation between the modelled pit shell outline and the PAF material was considered to be an important consideration when fine tuning pit optimisation as drill and blast activities would have the potential to compromise PAF material below the pit floor. Consequences may include atmospheric and/or aqueous oxygen ingress thereby initiating the oxidation of reactive sulfides and subsequent AMD generation. Clearly, a better understanding of the location of any PAF material can assist with optimising mine planning, and reduce the potential for AMD generation; commensurate with a cost/benefit assessment of managing that risk as against ore exploitation.

To better visualise the spatial distribution of PAF material, a 3D geochemical block model was constructed using the Discover 3D software package.

The 3D geochemical modelling was constructed by:

- Importing the pit shell data directly from the resource model.

- Importing all assay and drill hole data available from exploration drilling to a lateral distance of up to $50 \mathrm{~m}$ from the proposed pit shell to incorporate any low grade cut material ultimately reporting as mine waste. 
- Importing the geological surfaces from the Vulcan geological model to ensure accurate lithological boundaries were used.

- Net acid production potential (NAPP) values were calculated for all assay data points within the model (being maximum potential acidity as determined conservatively using total sulphur data minus acid neutralising potential as also conservatively determined utilising total calcium assay data). As a first pass for PAF risk, this proved to be a useful strategy.

The resultant 3D model produced was used to create a contoured risk map showing the modelled distance between the pit shell and the geological surface of the McRae shale unit (Figure 3).

From the 3D modelling, the cross sections as shown in Figure 4 was able to show schematically that the PAF material was located exclusively below the base of the pit shell. Furthermore, all materials within the pit shell appear to be non-PAF material based on the desktop assessment. The cross section shown in Figure 4 also indicates that PAF materials are unlikely to be found in the upper McRae shale unit. This is an important observation given that risk mapping indicated the upper McRae shale unit may be selectively exposed in the pit floor/walls.

There are clear benefits of using the 3D geochemical modelling package, even at desktop stage, primarily being the ability to visually conceptualise the marriage of geochemical, geological, and mine planning data. This visual feedback subsequently elevates areas of AMD risk, whereby additional work can be prioritised; ensuring the best return on investment.

Information gleaned as a result of the 3D geochemical modelling utilising desktop data included:

- That all materials within the pit shell were unlikely to be PAF based on the data assessed to date.

- That PAF materials identified based on the data assessed to date were located greater than $10 \mathrm{~m}$ below the modelled pit base.

Additional, ongoing geochemical data will be fed into the model to validate the findings and/or amend the pit optimisation/materials handling and storage strategy accordingly.

\subsection{Case study 2 - Pilbara, WA}

A similar study utilising similar methodology was undertaken for a second greenfields iron ore mine located in the Pilbara in WA. The desktop AMD risk assessment this time however, also utilising conservative NAPP calculations using assay data from the geological database indicated that sulfur bearing minerals were potentially present within the modelled pit shell. The exact spatial extent of this PAF material had not been established as exploration drilling data was still being input into mine models. Based on the results of the desk study assessment, 3D geochemical modelling using Discover 3D was again undertaken as per the methodology described for Case Study 1, above.

Five additional boreholes were commissioned under the infill drilling programme to obtain additional geochemical data based on a targeted approach utilising the 3D modelling output.

Figure 5 presents the model output, PAF material is indicated by net acid producing potential (NAPP) being greater than $10 \mathrm{~kg} / \mathrm{t} \mathrm{H}_{2} \mathrm{SO}_{4}$.

Figure 5A shows the occurance of PAF material, and Figure 5B shows a slice through the model showing all calsses of material (green indicates non acid forming material as the NAPP values is less than $0 \mathrm{~kg} / \mathrm{t} \mathrm{H}_{2} \mathrm{SO}_{4}$. 


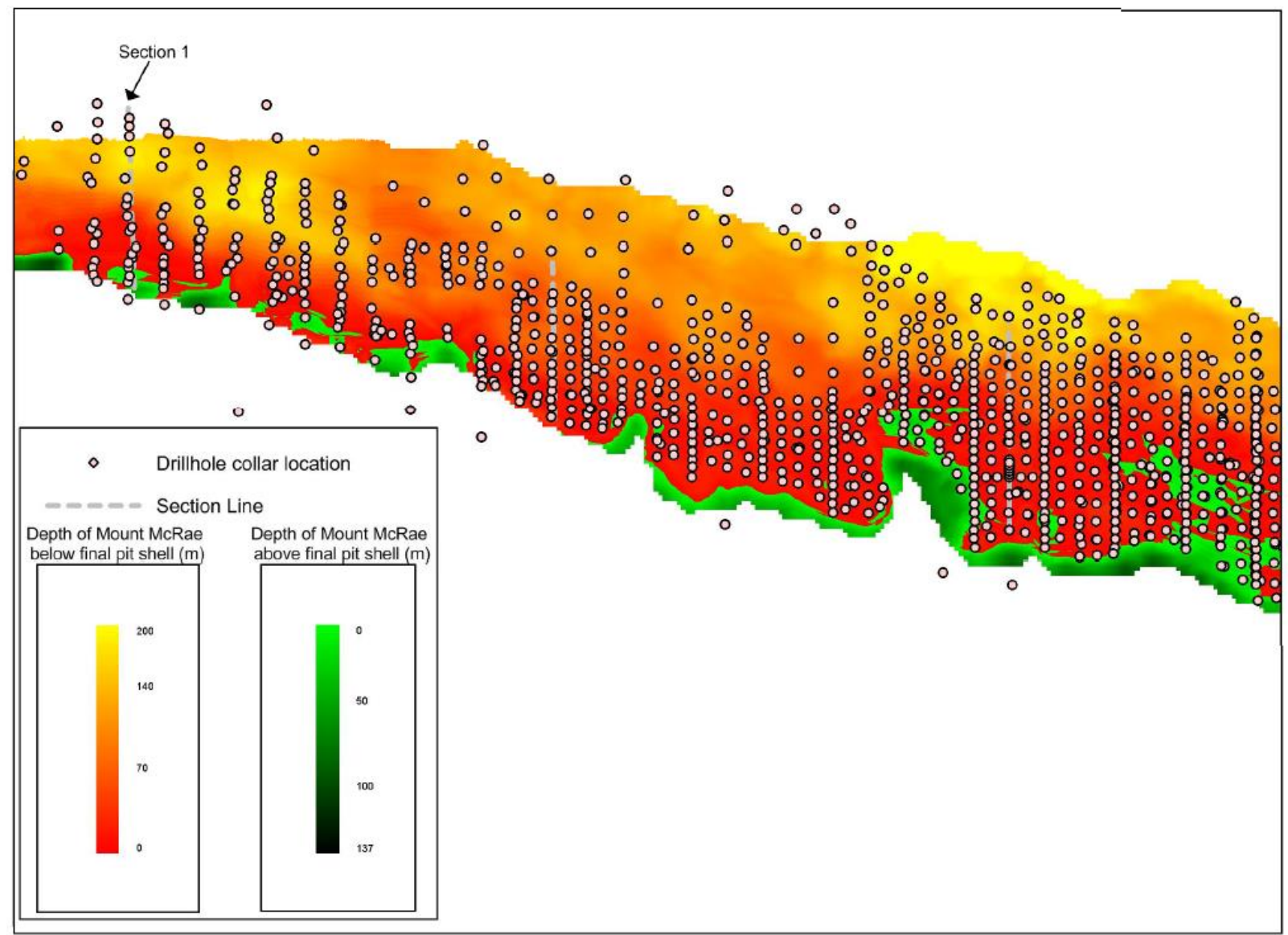

Figure 3 3D geochemical model output showing distance between pit shell and McRae shale Unit surface

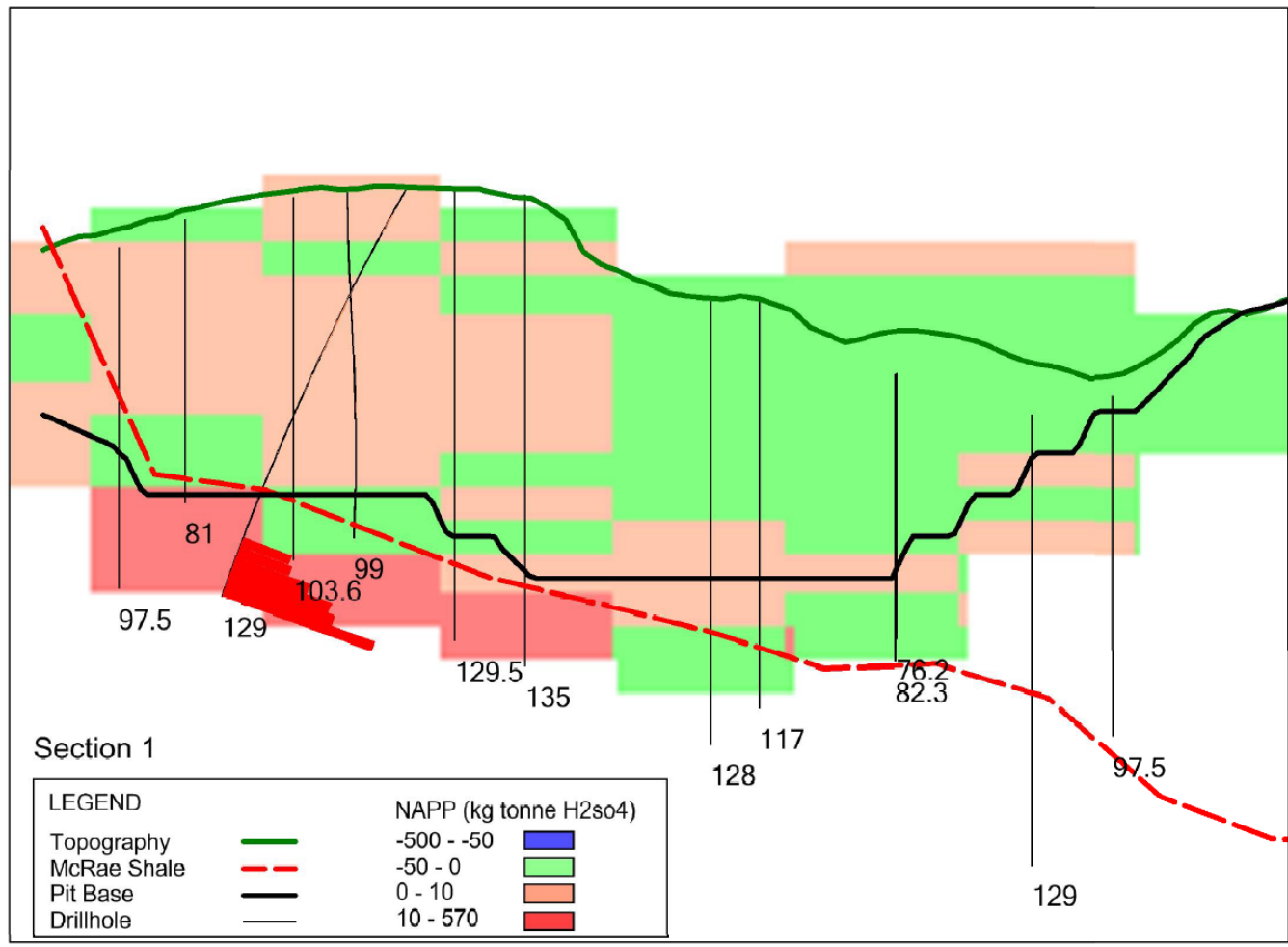

Figure 4 3D geochemical model output showing McRae shale surface, PAF material, and pit shell 

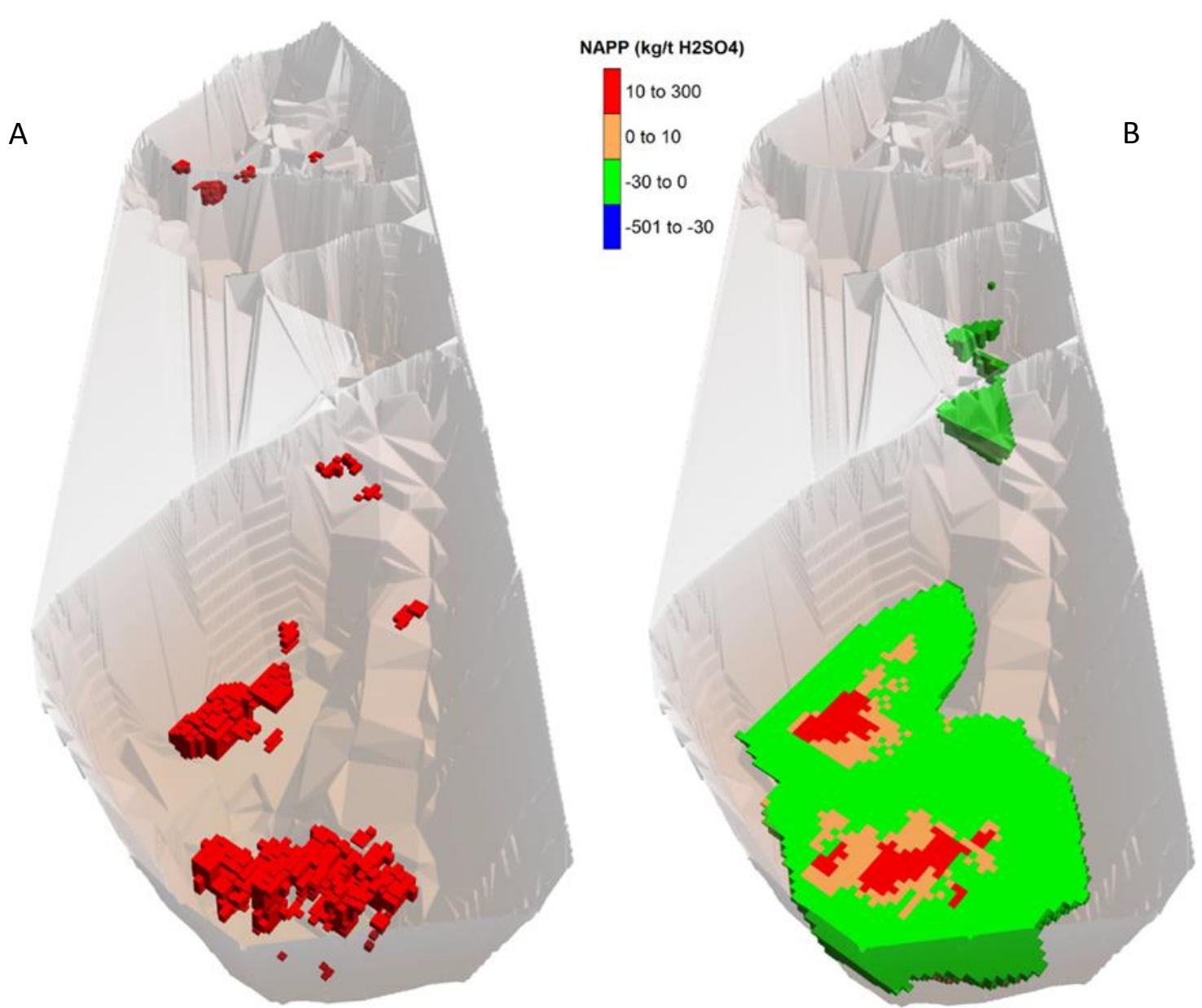

\section{Figure 5 3D geochemical model showing PAF material within the pit shell}

Whilst the concept of iterative collaboration between project teams is an idealised scenario, it takes some level of forward planning to avoid potential limitations. For example, during this case study, it eventuated that the geological and geochemical datasets did not directly correlate at some spatial locations. This was subsequently assessed and attributed to micro-scale lithological heterogeneity which was not reflected in the geological model which grouped areas into the dominant lithological class by cell. However, that consideration notwithstanding, the 3D geochemical visualisation model still allowed the PAF material to be mapped, and subsequent correlation of PAF material against lithological unit.

The 3D geochemical model allowed for early appraisal of AMD risk based on the visual representation of in pit PAF material. These data were subsequently included in the pit optimisation process via risk workshops. Importantly, areas of PAF material were also identified outside the pit shell, which provided valuable input for management of dewatering risk throughout the life of the mine. In examples such as this one whereby mine scheduling occurs following mine waste desktop assessment has been completed, 3D geochemical modelling may be used to provide spatial assessment of PAF material. The data has been utilised in preliminary pit optimisation modelling, with the PAF material and its location being factored into mine scheduling and subsequent selective handling and placement. The model is of course dynamic and will be built upon pending additional geochemical and geological data to enable further pit optimisation modelling to be undertaken. Such data would also be utilised in mine waste transport and placement modelling from a cost minimisation perspective, in addition to minimising AMD risk.

\subsection{Case study 3 - Peru}

Mine planning activities were undertaken for an open pit gold/copper operation in sub-tropical northern Peru. The final configuration of the project planning is an example of how the different and disparate 
disciplines can come together to successfully plan a mine. This project has significant AMD implications with respect to its' initial permitting, ongoing operations and post closure behaviour.

Physically, the project was in challenging physical terrain, and a complex planning and operating environment, such that:

1. There was a general lack of 'competent' waste materials on-site from which to construct the tailings storage facility (TSF). The TSF could not be completely pre-constructed. Its construction had to be an on-going project throughout the mine life. The successful and timely construction of successive lifts on the TSF controlled the Mills' output.

2. The general Run of Mine waste had significant potential to generate AMD.

3. The waste dumps, and the TSF, were to be constructed on medium to severe topography. Therefore all the waste destinations had to be considered an 'engineered' structure, rather than just a waste dump.

4. Terrain meant that there was limited area for stockpiling materials; however the ore types meant that campaign milling needed to be used. Therefore oxide ores were stockpiled during sulphide ore mill campaigns, and vice versa.

The key outcome of the project was an integrated approach to the development of the mine planning process that incorporated geochemical assessment with mine design and planning that:

1. Correctly characterised the waste types as to their engineering properties and their geochemical properties. Of concern was the presence of significant volumes of Non Acid Forming (NAF) Waste - that was quite incompetent from an engineering perspective. Hence it could not all be used as a generic inert capping material. The characterisation required was not only of the source material. The source material was characterised in the pit geology model based on geochemical assessment as described in the previous case study. But also the Waste destination and TSF cells were characterised as to what type of waste could be deposited into each cell - which was more closely a 'basis of design' type assessment. Hence both the source and the destination needed characterisation.

2. Brought the waste destinations and the TSF into the same planning environment into which the pit was being planned. Therefore true 'source/destination' planning could be achieved. We showed what blocks came from where in the pit, and where each waste block had to go into the TSF or waste destination. Effectively when a block of material was 'sourced' from the pit, a conforming destination where that material could enter was uniquely selected as its destination.

3. Demonstrated that insufficient waste materials of the correct characteristics could be sourced, in a timely manner, directly from the pit. Therefore the successful pit operation also had to incorporate the timely planning of outlying limestone quarries.

4. Showed how compliance to AMD and Engineering requirements, amongst other requirements, could be achieved - in the one mine plan. That is all of the planning requirements, from the diverse stakeholders, were shown to be demonstrated. Significant value and agreement was obtained in having a single mine plan circulated, rather than different versions of a mine plan. It is worth noting that this was an iterative process.

5. Showed the timing and resources required to achieve this mine plan. For example what equipment hours need to be assigned to which activities, in different areas of the mine operations, in each period - to achieve a satisfactory outcome for all? This outcome, in turn, formed the basis for the contractual agreement with the mining contractor. In this way it has been translated into an operational plan. 


\section{$7 \quad$ Summary and conclusions}

AMD remains arguably one of the greatest impediments to the mining industry realising environmental sustainability. Today's taxpayers are currently funding mining environmental legacies dating back over a century. Fortunately, the global AMD knowledge base is growing, thanks to an increasingly coordinated approach. That fact notwithstanding, increasingly specific regulatory requirements that ultimately reflect community expectations are requiring mining proponents to undertake increasingly rigorous AMD risk assessments prior to mines being approved. The fundamental mechanism to gain input into the AMD risk assessment is a mine waste geochemical assessment.

This paper attempted to demonstrate the cumulative strengths of employing an integrated approach to AMD assessment by ensuring that the three key teams at the mine scoping and/or pre-feasibility stage engage in iterative knowledge transfer. The integration of information from exploration geology, mine planning and environment/geochemical characterisation ultimately ensures that longer term AMD risk is minimised.

Whilst not derived in this paper, there is arguably a clear business case for ensuring AMD risk is minimised throughout the life of mine and beyond by integrating the knowledge base between teams during the early stages of mine planning. Indeed, whilst saving money through optimising pit design and materials handling and placement, thereby minimising any need for double handling; ultimately, many regulators today will not approve a mine without a conceptual mine closure plan. Increasingly, this level of detail expected within these mine closure plans, even at approvals stage, is increasing.

The three examples utilised herein referred to an application of mine waste geochemical assessment whereby AMD risk must be considered utilising geochemical characterisation. The two WA examples herein have shown that the geochemical dataset, even at a desktop first pass level, can be fed into a 3D software model that integrates with the geological block model, ultimately enabling the mine planners to iteratively optimise pit design as additional geochemical and geological data is added.

Such a simple, yet effective, advancement can only reduce risk if utilised effectively; thereby reducing cost, and increasing industry credibility by reducing long term AMD risk through effective mine waste management and long term placement.

\section{References}

Brundtland, G. (ed) (1987) Our common future: The world commission on environment and development, Oxford University Press, Oxford, England.

DITR (2007) Department of Industry Tourism and Resources, Managing acid and metalliferous drainage, leading practice sustainable development program for the mining industry, Canberra, Australia, $107 \mathrm{p}$.

Harries, J. (1997) Acid mine drainage in Australia: Its extent and potential future liability, Supervising Scientist Report 125, Supervising Scientist, Canberra, $94 \mathrm{p}$.

ICMM (2003) International Council on Mining and Metals, Sustainable Development Framework, Available at www.icmm.com.

INAP (2009) International Network for Acid Prevention, Global Acid Rock Drainage Guide (GARD), Available at: http://www.gardguide.com/index.php/Main_Page.

Mudder, T. (2004) Coming down hard on ARD, Mining Environmental Management, May 2004, p. 2.

Western Australian Department of Mines and Petroleum and the Western Australian Environmental Protection Authority (2011). Guidelines for preparing mine closure plans, Perth, $78 \mathrm{p}$. 
Managing closure risks by integrating acid and metalliferous drainage assessments

S.R. Pearce et al. with mine scheduling - real world applications 